\title{
Des histoires tristes
}

\section{Dominique Goy-Blanquet}

\section{(2) OpenEdition \\ Journals}

Édition électronique

URL : http://journals.openedition.org/shakespeare/492

DOI : 10.4000/shakespeare.492

ISSN : 2271-6424

Éditeur

Société Française Shakespeare

Édition imprimée

Date de publication : 1 novembre 1984

Pagination : 31-50

Référence électronique

Dominique Goy-Blanquet, «Des histoires tristes », Actes des congrès de la Société française Shakespeare [En ligne], 5 | 1984, mis en ligne le 01 janvier 2007, consulté le 22 avril 2019. URL : http:// journals.openedition.org/shakespeare/492 ; DOI : 10.4000/shakespeare.492 
SOCIETE FRANÇAISE SHAKESPEARE

Actes du Congrès 1983

\section{MYTHE ET HISTOIRE}

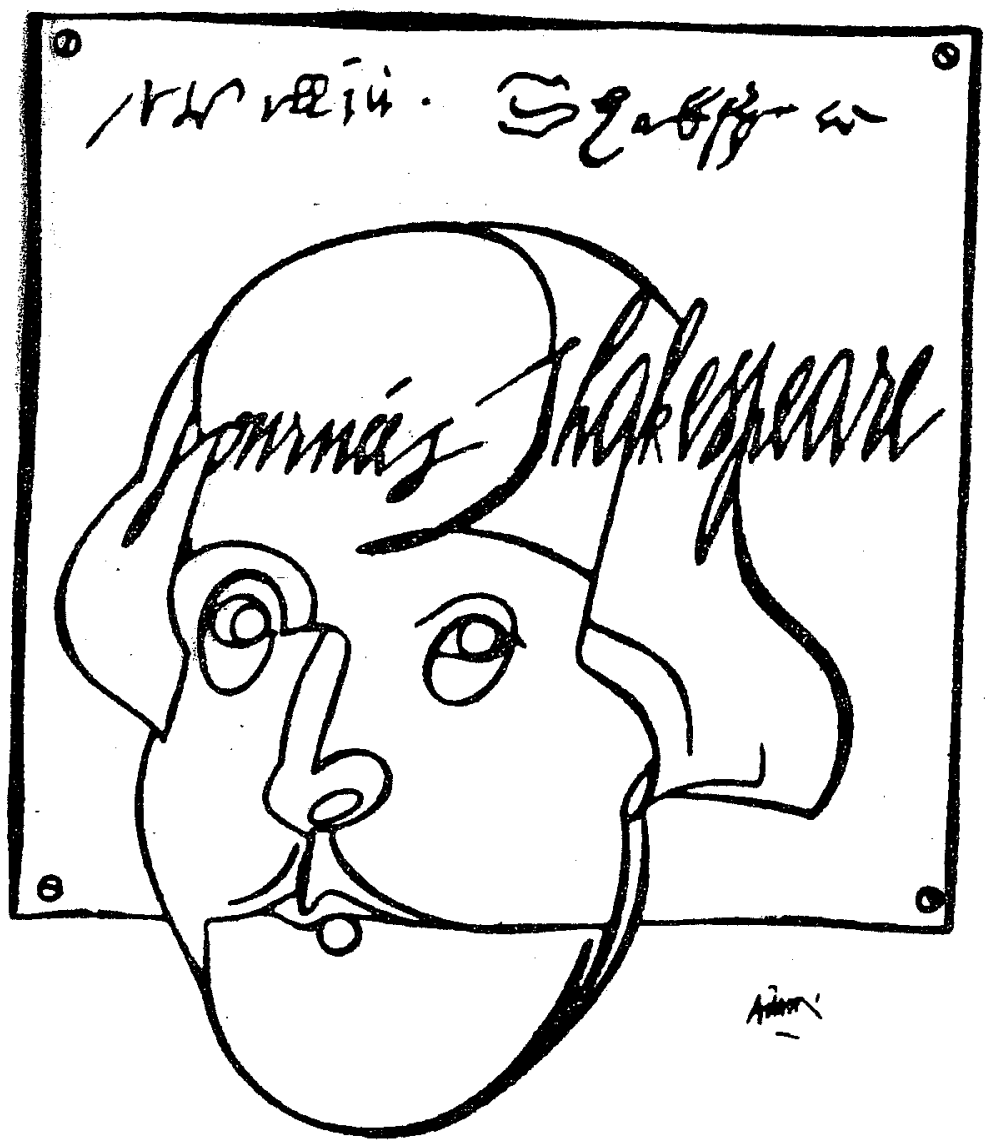

$$
\begin{array}{r}
\text { DiReCteur DE LA' PUblicatic } \\
\text { M.T. Jones-Davie }
\end{array}
$$

Publié avec le concours du Centre National de la Recherche Scientifiq

$$
\text { JEAN TOUZOT Libraire - Editeur }
$$

38, rue Saint-Sulpice 75278 PARIS CEDEX 06199 


\title{
DES HISTOIRES TRISTES
}

\author{
«Adam et Eve, c'était avant \\ ou après les dinosaures?» \\ Marie BLANQUET.
}

A la Renaissance, l'histoire était reconnue d'utilité publique, et multiple. Vue du $\mathrm{XX}^{\grave{e}}$ siècle, pratiquement toute la production à caractère historique de la période Tudor est au moins en partie mythique au sens moderne du terme, légende ou falsification délibérée. A une époque en mal de légitimité, l'industrie des mythes de fondation était des plus actives : la famille royale donnait l'exemple en s'inventant des origines légendaires grâce à une généalogie fantaisiste, et à sa suite le Parlement, les institutions, l'Eglise et les simples particuliers se fabriquaient des pedigrees. L'autorité, et la respectabilité, s'appuyaient sur l'ancienneté immémoriale des origines ; les petits bourgeois se contentaient de remonter jusqu'à la conqué te normande, mais la famille Tudor et le Parlement s'estimaient antérieurs à la fondation de Rome.

Tous les usagers de l'histoire proclamaient qu'elle était au service de la vérité, avant de lui faire rendre une quantité d'autres services. La recherche de la vérité était rarement une quête désintéressée :

A catalogue of what history taught would include the following : morals, manners, prudence, patriotism, statecraft, virtue, religion, wisdom, truth ; history was equally an antidote to all opposite qualities. History could have tamed the Irish, and history was a salutary remedy for mental sickness. Knowledge of history helped one to rise in the world, [. . . The publicity value of history was well understood,$[\ldots]$ 
by projectors and preachers ; and history's value as propaganda was firmly grasped by Court, Parliament, and Convocation. The uses of history were almost as various as the understandings of men. ${ }^{1}$

Par ailleurs, l'histoire remplissait d'autant mieux ses diverses fonctions qu'elle se présentait sous une forme littéraire harmonieuse et plaisante, c'est-à-dire qu'elle usait des ressources de l'art pour atteindre son but, instruire, édifier, convaincre :

In poems and plays and histories authors sought to delight and to instruct their audiences by telling true stories, that is, histories. Whether or not such history was true to the evidence, it had to be true to the literary purposes of the author, that is , true to his moral or patriotic theme, or simply poetically universal in Aristotle's sense - ${ }^{2}$

Cette tendance généralisée du mythe à absorber l'histoire n'était pas toujours le fait d'une falsification délibérée. Il est fréquent que les récits mythiques qui relatent l'origine légendaire, magique ou divine, d'un groupe humain incorporent des événements particuliers de son histoire en les faisant glisser dans des catégories archétypales. Il semble alors que la mémoire collective enregistre le fait individuel pour ce qu'il a d'universel et d'exemplaire, en le dépouillant de ses particularités historiques; ce n’est pas l'événement qu'on retient, mais le récit qu'on en fait :

As time goes on, and historical tradition becomes more tenuous, only the events with conventional poetic associations can carry the thrilling magic of a great name. ${ }^{3}$

Les faits ne s'inscrivent dans la mémoire qu'avec un certain 
effet de mythisation, ils prennent une forme assimilable en s'insérant dans un récit constitué. Le mythe est également fondateur de tradition littéraire ${ }^{4}$.

L'exemplarité du mythe a souvent pour effet de moraliser l'histoire. Une fois détachés de leur contexte, les événements du passé inspirent des fables édifiantes, valables pour l'éternité. Les innombrables bienfaits de l'histoire sous les Tudors correspondent à peu près à trois types d'usage : le profit, la morale, et la polémique. Tous trois postulent l'analogie entre le présent et le passé, et d'une manière générale la supériorité de l'ancien sur le nouveau; les leçons de l'histoire ont valeur universelle, et l'autorité s'appuie sur le précédent et la coutume. Quant à savoir si les pièces de Shakespeare sont plus ou moins mythiques que l'ensemble de l'historiographie contemporaine, il faudrait d'abord préciser de quel my the on parle.

Le champ des définitions est vaste, de la mythologie comparatiste à l'anthropologie structurale. Le mythe, apprend-on, est un récit des origines, un récit qui dit quelque chose sur le Cosmos, sur l'origine de l'homme et l'origine des dieux ${ }^{5}$. Le contenu des mythes n'a pratiquement rien de vraisemblable mais il est reconnu pour vrai par les sociétés qui les racontent. Ces récits, qui se ressemblent d'un bout à l'autre de la terre, ont toujours une structure cohérente qui permet toute une série de variantes combinatoires ; le mythe est un modele logique pour résoudre une contradiction ${ }^{6}$. Leur cohérence logique fournit les archétypes qui sont les matrices des formes littéraires : l'archétype des archétypes - le dieu qui meurt et qui renait, le grand cycle de la nature - est la colonne vertébrale de toute la littérature ${ }^{7}$.

Les mythes sont l'expression structurée d'un code symbolique ; ce code est «un langage qui solidarise la personne humaine, d'une part avec le Cosmos et de l'autre avec la Communauté dont il fait partie». Le mythe remplit la double fonction du symbole : 1) fonction hiérophanique - assurer le contact avec le divin; 2) résolution des tensions - réaliser l'harmonie de la société. A la fois «exprimer en termes concrets et familiers ce qui est caché et 
imprévisible » et aider ainsi l'homme à domestiquer « les forces sauvages et capricieuses de la nature ${ }^{8}$.

Entre ces deux pôles se situent les nombreux usages qu'on fait du récit mythique : magique, thérapeutique, cérémoniel, politique ... La force du récit est double puisque simultanément il interprète et il réactive l'événement primordial ; Claude Levi-Strauss compare l'effet du récit mythique à celui d'une cure psychanalytique ${ }^{9}$.

Le mythe peut justifier, légitimer, guérir. Mais bien sûr il ne remplit ses diverses fonctions qu'autant qu'il obtient l'adhésion des auditeurs : ce que nous appelons mythe, ce sont toujours les croyances des autres.

Il est probable que Shakespeare partageait un grand nombre des préjugés et des croyances de ses contemporains. Toutefois il ne sera pas question ici des mythes «reconnus pour vrais»à son époque, mais de ce que lui-même aurait pu désigner comme my the, c'est-à-dire les croyances des autres, justement celles qui sont remises en question dans ce qu'on appelle les deux tétralogies historiques, les huit pièces qui sont consacrées aux derniers Plantagenets. En effet, on peut se demander pourquoi Shakespeare a écrit des pièces historiques, pourquoi il a choisi cette période particulière, et pourquoi il n'a pas suivi l'ordre chronologique de ses sources.

Ces huit pièces ne sont pas exclusivement des enquêtes historiques sur le passé de son époque.Tout d'abord ce sont des mythes au sens où l'entendait Aristote : dans sa théorie, le muthos désigne à la fois l'agencement des faits dans l'oeuvre dramatique et le mythe qui en est la substance narrative, mais l'opposition entre mythe et histoire n'est pas pertinente ; le théâtre tragique grec s'inspire du passé plus ou moins légendaire de la cité, son histoire mythique, il ne se qualifie pas par l'authenticité des faits représentés mais par son aptitude à produire sur le public l'effet propre de la tragédie : éveiller les sentiments - de frayeur et de pitié, purifiés par l'alchimie mimétique. Et Aristote recommande aux poètes en quête d'histoires : "le surgissement de violences au coeurdes alliances [...] Voilà ce qu'il faut rechercher.» ${ }^{10}$ Tout en reconnaissant 
qu'il n'y a guère dans l'histoire de la cité que quelques familles qui satisfont cette exigence :

comme ce n'est pas à la connaissance de l'art, mais au hasard, que les poètes doivent d'avoir, en cherchant, trouvé de telles combinaisons pour leurs histoires, ils sont forcés de retomber sur les maisons auxquelles est échu ce genre de violences $^{11}$.

Ainsi ce que vont chercher les poètes dans les récits du passé, ce sont avant tout de belles histoires, des histoires qui font peur et qui font pleurer. Aristote n'établit pas de distinction entre les personnages historiques et les personnages épiques ou légendaires, il n'exige pas qu'ils soient conformes à la vérité ou à la tradition littéraire, mais qu'ils soient convaincants.

Pour le public d'Eschyle, l'histoire des Atrides appartenait à un passé lointain mais toujours vivant. Et pour les élisabéthains, les tribulations des Plantagenets avaient ouvert assez de crises dont les traces étaient encore sensibles pour éveiller des échos dans toutes les mémoires. Shakespeare a trouvé dans les chroniques la matière d'une série d'histoires tristes et terribles : une vaste geste familiale, développée sur plusieurs générations, assez riche en conflits violents pour combler Aristote. L'événement crucial, dans ces épisodes, c'est la mise à mort du roi, qui n'est pas un fait rare dans l'histoire anglaise, mais qui atteint quand même une fréquence particulièrement élevée au cours de cette période. Un événement à forte résonance mythique, de même que les tentatives du chroniqueur pour accommoder les incohérences. de l'histoire à une intervention surnaturelle. La chronique offrait aussi un nom, d'une grande fertilité onomastique, Plantagenet, et un emblème, un soleil rayonnant.

Certains épisodes historiques sont bien de nature à émouvoir, mais ils n'atteignent pleinement leur but qu'après avoir été mis en forme par l'art du conteur ; comme Shakespeare le suggère à plusieurs reprises, c'est 
le passage par la narration qui émeut par ricochet les témoins de l'événement, au moment, comme dirait Northrop Frye, où s'instaure une tradition littéraire ${ }^{12}$. Avant d'être mis à mort, le duc d'York se voit offrir un mouchoir trempé dans le sang de son fils pour sécher ses pleurs, et en évoquant le récit qu'on fera plus tard de ses souffrances, il tire des larmes à ses ennemis (3H6, I. iv. 159-164). Richard II, quand on le sépare de sa femme, lui confie le soin de raconter son histoire :

In winter's tedious nights sit by the fire With good old folks, and let them tell thee tales Of woeful ages long ago betid; And ere thou bid good night, to quite their griefs Tell thou the lamentable tale of me, And send the hearers weeping to their beds $[\ldots]$

For the deposing of a rightful king. $(R 2$, V. i. $40-50)$

Par l'entremise de Richard, Shakespeare invite le public à s'asseoir pour écouter les histoires tristes de la mort des rois. C'est autour de cet événement capital que s'organisent les deux groupes de pièces : c'est le point d'aboutissement de la première série - après exécution de trois têtes couronnées - et le point de départ de la seconde. Les pièces où on tue le roi-3 Henry VI, Richard III, Richard II - déploient les éléments mythogènes de la chronique en les associant à l'acte crucial.

La mise à mort du roi, et sa relation avec le mythe du dieu qui meurt, est un archétype qui informe un grand nombre de rites sacrificiels primitifs, et une multitude d'oeuvres littéraires. En étudiant les origines magiques de la royauté, James G. Frazer a mis en évidence les éléments sacrés qui accompagnent l'exercice du pouvoir souverain dans les cultures les plus variées ${ }^{13}$, et tout en remettant sérieusement en question ses méthodes d'analyse, les anthropologues modernes confirment que ces éléments se retrouvent de manière assez constante, indépendamment 
des situations historiques particulières. Une série d'études comparatistes sur le Pouvoir et le Sacré les résume ainsi :

- la place du roi à la tête de certains rites de conservation de la vie et de la fécondité

- les rites de meurtre royal indispensables à la transmission du pouvoir

- les interdits pesant sur l'approche de la personne du souverain ${ }^{14}$.

De par les pouvoirs mystérieux qu'on lui prête, le roi se retrouve régulièrement associé au cycle de la végétation, à la régularité saisonnière ; il est responsable de la fertilité du sol et de la survie de la communauté. Frazer pense que ces rois ont été à l'origine des sorciers, guérisseurs ou faiseurs de pluie, qui ont acquis le pouvoir supréme en vertu de leurs dons magiques, avec cette contrepartie logique, que si ça va mal, et qu'il se révèle incapable de remplir ses fonctions, on se débarrasse du roi.

En fait la relation entre la conjoncture mauvaise et l'exécution du monarque n'est pas aussi simple et automatique. Il y a toutes sortes de bonnes raisons de tuer le roi, et l'exécution va du meurtre pur et simple, et définitif, à la cérémonie répétée à intervalles réguliers, qui simule sa mise à mort et sa résurrection. Selon les coutumes, on tue le roi parce qu'il est incapable - il ne sait pas faire tomber la pluie - ou malfaisant - il pourrait mais il ne veut pas, c'est lui qui l'empêche de tomber-, parce qu'il vieillit ou qu'il est malade, et il pourrait communiquer cet état de faiblesse à son environnement : dans ce cas on le tue aux premiers signes de vieillissement, à moins qu'on n'ait fixé au préalable le nombre d'années de son règne, au terme duquel il doit être exécuté. Ou encore il faut que son successeur le tue pour prendre sa place, et c'est l'épreuve indispensable à la transmission du pouvoir. Ou bien sa mort marque la fin d'une civilisation jugée fruste et archaïque, il doit disparaitre pour permettre l'avènement d'une société nouvelle ${ }^{15}$.

La mise à mort peut être un sacrifice offert pour apaiser ou séduire les puissances supérieures : de par sa double nature, humaine et divine, le roi est un médiateur 
entre la terre et le ciel.

Enfin la mise à mort peut être simulée dans des cérémonies annuelles qui représentent et réactivent le mythe solaire. Par sa mise à mort symbolique le roi incarne le dieu qui meurt et qui renaît après un passage dans le monde souterrain, pour garantir la régularité saisonnière et le renouvellement de la végétation : un rite de restauration destiné à expulser le mal accumulé au cours de l'année, effacer l'usure du temps, et favoriser l'action des forces de renouveau dans le monde naturel ${ }^{16}$.

$\mathrm{Ce}$ qu'il y a de commun à toutes ces pratiques, c'est qu'elles satisfont à l'idéologie des trois fonctions qui, d'après Dumézil, jouent un rôle directeur dans la plupart des mythes parce qu'elles correspondent à trois besoins que tout groupe humain doit satisfaire pour ne pas périr : la royauté magique et juridique, la force physique, et la fertilité ${ }^{17}$.

Henry VI et Richard II sont persuadés du caractère sacré de la monarchie. Tous deux rappellent à leurs sujets inconséquents que l'onction divine a laissé sur eux une marque indélébile, et ils prennent le ciel à témoin de l'impensable sacrilège commis contre eux. Ils représentent la conception chrétienne médiévale de la monarchie : le roi est l'oint du Seigneur, l'incarnation vivante de la volonté divine à laquelle toutes choses sont soumises. Les références bibliques sont nombreuses et explicites : la fin de Richard III renvoie à l'Apocalypse, celle de Richard II à la chute d'Adam ; mais elles n'en sont pas des allégories. Les textes bibliques sont là, dit Edward I. Berry, comme un mythe culturel sous-jacent, une présence archétypale qui relie la pièce aux structures plus larges de l'histoire chrétienne ${ }^{18}$.

Egalement sensibles, mais en partie voilés par la conception chrétienne de la monarchie sacrée, on découvre des éléments plus archaïques qui rapprochent les rois de Shakespeare de ces rois mythiques qu'on met à mort pour garantir la survie de la communauté. Dans les pièces où on sacrifie le roi, la trajectoire des personnages royaux est en association étroite avec le cycle de la végétation, les 
mythes solaires, et les rythmes saisonniers.

Il n'est pas rare dans le théâtre de Shakespeare que la tempête se déchaîne en même temps que les passions, et que le monde naturel retentisse de tous les désordres humains. Cette solidarité de la création est bien plus qu'une métaphore pour les élisabéthains, et elle est sensible dans l'ensemble des pièces historiques.

Dans 3 Henry VI, le désordre qui ravage le royaume se traduit par l'anarchie ambiante et la perturbation des rythmes naturels. La crise du pouvoir a des effets chaotiques : la division de l'astre solaire, une agriculture sanglante, et le bouleversement des saisons.

Le soleil est divisé parce qu'il y a deux prétendants au trône, identifiés à Phoebus et Phaëton qui à eux deux ont mis le feu à la terre (I. iv. 33, II. vi. 10-13). Le duc d'York, Phaëton tombé de son char, disparaît pour renaître en son fils Edward. Tandis qu'il ignore encore la mort de son père, Edward voit le soleil se diviser en trois astres, qu'il compare aux trois fils d'York, puis n'en faire qu'un seul, et prenant cette vision pour un heureux présage, il décide d'en faire son emblème (II. i. 25-40). Le soleil d'Edward est deux fois caché par les nuages ennemis avant de triompher (II. iii. 7, V. iii. 1-13), mais il ressurgit et précipite Icare - le jeune prince de Galles - dans la mer jalouse (V. vi. 21-25).

$\mathrm{La}$ course erratique du soleil dérègle l'écoulement du temps : le jour se bat contre la nuit (II. v. 1-2) ; le printemps est amputé par l'hiver (II. iii. 46-47), l'été aboli (IV. viii. 60-61), et c'est en automne que les vainqueurs rentrent leur moisson (V. vii. 3-4).

Le blé d'automne de la famille d'York, ce sont les têtes de leurs ennemis fauchés sur le champ de bataille (V. vii. 3-20). Depuis la proclamation orgueilleuse de Warwick au début de la pièce, "I'll plant Plantagenet" (l. i. 48), tous les travaux des champs se font à la hache : la hache tranche les rameaux et la souche des arbres généalogiques, souches dynastiques et rameaux princiers (II. vi. 46-51, II. ii. 163-169, III. ii. 126) ; elle ouvre un chemin dans les ronces qui s'opposent aux désirs ambi- 
tieux (III. ii. 174-181), déboise des armées entières (V. iv. 67-7.1), plante et déracine les rois (I. i. 48, III. iii. 198, V.v. 48).

Henry VI, le jardinier trop faible qui a laissé prospérer les herbes folles (II. vi. 21), rappelle à la mémoire tout ce que la guerre a détruit : il évoque la simplicité biblique de la vie pastorale, idéalement harmonisée au rythme de la nature, qui accomplit les desseins du Créateur sans brutaliser les saisons (II. v. 42-54). Responsable de l'ordre naturel, il offre sa vie en sacrifice, une rose pour en sauver mille (II. v. 94-102), mais son immolation ne suffit pas à rétablir l'orđre.

Désormais la terre est ivre de sang humain (II. iii. 15-23) et elle a accouché d'un monstre difforme, désavoué par la Nature, dont le dos est comme une montagne, le bras un buisson flétri, «like to a chaos» (III. ii. 153162), qui ne ressemble à père ni mère (II. ii. 135, III. ii. 162). Une fois rentrée la moisson d'automne, le «radieux été» de Richard s'installe au coeur de 1 'hiver $(R 3$, I. i. 1-2), et les perturbations suivent leur cours jusqu'à la purification finale. Le soleil de Richard est caché, celui de Richmond rayonne (V. iii. 279-280, V. iii. 19). Richmond se propose de restaurer la paix par une dernière moisson sanglante, comme le moissonneur de l'Apocalypse (V. ii. 7-16). L'expulsion du mal, concentré en Richard, recrée l'unité de l'astre solaire, et reconsacre la terre meurtrie à sa destination première (V. v. 20-41).

Mais entre la course immobile du temps évoquée par Henry VI, la ronde des heures, et le temps dévorant de Richard, quelque chose s'est rompu, et il n'y a pas de retour posșible à l'ordre primitif, à ce temps primordial où le pouvoir royal faisait partie intégrante de l'ordre naturel. Cette harmonie idéale a été détruite par l'irruption de l'histoire, par la montée de forces nouvelles qui sont perceptibles dès la première partie de Henry VI et que le roi est bien incapable de contrôler. Ses nobles sont toujours farcis de légendes hérö̈ques, mais leurs ambitions n'ont plus rien de chevaleresque.

En fait Henry VI et Richard III sont complémentaires. 
En s'offrant en victime expiatoire, Henry VI prend sur lui la faute collective, mais il n'accomplit qu'une moitié de sa tâche ; l'autre, qui lui imposerait de contenir la violence par la violence, c'est la face d'ombre du pouvoir, celle qu'il se refuse à assumer. Cette violence incontrôlée enclenche un processus qui aboutit à Richard, le bouc émissaire.

Si l'excès de vertu d'Henry VI produit l'excès de crime de Richard, il est clair que tous deux doivent disparaître pour faire place à un nouveau régime capable de prévenir un tel ravage. L'ordre nouveau s'incarne en Richmond, qui est le grand inconnu de la pièce. Il arrive à la fin comme l'enfant du miracle, pour fonder une société idéale, dont on ne sait rien. Personne ne vient expliquer comment il va s'y prendre pour concilier les devoirs du chrétien et ceux de l'homme d'Etat, comment il va réussir là où même le sage Gloucester avait échoué. Les questions sont suspendues, il faut croire que maintenant tout ira bien.

Comme disait Sir Walter Ralegh, si l'historien serre la vérité de trop près, il risque de prendre des coups de pied dans les gencives. Montrer la suite de Richard III, c'était mettre en cause la dynastie Tudor.

En fait la seconde tétralogie est bien la suite de la première, même si elle représente des événements antérieurs; elle reprend des questions qui étaient restées sans réponse, et qu'il aurait été difficile de traiter avec toute la liberté de manoeuvre voulue s'il s'était agi d'examiner de près les agissements de Richmond. Ces questions se posent dans King John, composé entre les séries York et Lancastre ; l'histoire d'Henry IV et d'Henry V s'y attaque de front. I1 est assez apparent que Shakespeare n'est pas remonté dans le temps pour éclairer les origines historiques de la Guerre des Roses, mais probablement pour poursuivre une réflexion qui a été bloquée par l'arrivée de Richmond, le premier Tudor.

La première tétralogie dramatise une crise de l'institution monarchique qui se traduit par cette contradiction :

- il est impensable de tuer le roi

- il est indispensable de tuer le roi

contradiction qui est neutralisée par une résolution magique 
du conflit en la personne de Richmond : la guerre devient la paix, les ennemis sont des amis, et le meurtre du roi un acte de salut public.

Le second groupe de pièces reprend avant l'acte crucial et repose le dilemme insoluble : il est impensable/ indispensable de tuer le roi, et que faire ensuite si on l'a tué quand même. Ou autrement dit, comment se règlent les comptes entre le pouvoir et le sacré, entre le mythe et l'histoire?

On retrouve dans Richard II les images de la course solaire et du cycle de la végétation. Dans la vision passéiste du vieux Gaunt, l'Angleterre est identifiée au jardin d'Eden, vision prolongée par la scène emblématique des jardiniers d'où il ressort que Richard II a bien mal cultivé son jardin.

La royauté est également liée au monde naturel par la métaphore de l'arbre généalogique, qui associe le sang royal au règne végétal. Richard II a tranché une des branches de l'arbre dynastique en faisant assassiner son oncle (I. ii. 11-21). Cependant il est le lieutenant de Dieu, «Anointed, crowned, planted many years, 》 (IV. i. 127); il y a grand danger à planter des rois illégitimes, qu'on peut arracher ensuite sans aucun scrupule, et Richard II décrit le mécanisme des rébellions qui vont troubler le règne d'Henry IV (V. i. 55-68). Les larmes de Richard vont dévaster les récoltes (III. iii. 161-163). Lui et son rival se menacent mutuellement d'arroser les pâturages d'une pluie de sang (III. iii. 42-50, III. iii. 95-100). Ce sang répandu engraissera le sol qui fera naître une moisson de guerriers, et la guerre civile désolera l'Angleterre pendant des générations (IV. i. 134-149).

De nouveau, deux soleils occupent en même temps le ciel, l'un monte, l'autre descend, tel Phaëton, et se confond parmi les ombres. Mais soucieux de ne pas renouveler les erreurs de Richard, Bolingbroke s'emploie énergiquement à éliminer chenilles et mauvaises herbes avant même d'avoir détrôné son rival (II. iii. 165-166). Ceci fait,l'astre ascendant ramène un nouveau printemps, ses nobles sont comme des violettes : c'est une modestie qui leur va bien, la prudence est recommandée si on ne veut pas se faire 
cueillir avant d'éclore (V. ii. 46-51). Pour finir, le sang de Richard arrose Bolingbroke de taches indélébiles, mais le nouveau roi avoue que ce sang est indispensable à sa croissance (V. vi. 38-46).

Richard II se demande comment il peut encore vivre après sa déposition, puisqu'il n'est rien s'il n'est pas roi (IV. i. 201-222). Après lui la scission est faite entre la majesté du pouvoir et la personne humaine qui le porte. Le mariage mystique du roi à son royaume est rompu tout comme le mariage de Richard avec son épouse. Il laisse son successeur aux prises avec sa moisson de guerriers, et la difficile entreprise de fonder le pouvoir en légitimité et d'en reconquérir la dimension sacrée. Pour reconstruire l'aura détruite, Henry IV s'isole et interdit qu'on approche de sa personne, ses apparitions en public sont organisées comme des cérémonies. Toutes ces mesures s'appliquent à dissimuler l'être humain derrière la pourpre royale.

La seconde série de pièces n'observe pas comme la première une progression historique rigoureuse. Dans les deux parties de Henry $I V$, le prince $\mathrm{Hal}$ se bat avec son difficile héritage. Il affronte une succession d'épreuves par lesquelles il conquiert effectivement le pouvoir, et par un système de réparations et de compensations, il lui restitue sa dimension mythique et fonde sa propre légitimité.

En prétendant au trône de France, «this best garden of the world» (V. ii. 36-37), il apaise le passé, l'Eden perdu de Gaunt, le jardin dévasté de Richard ; l'armée nationale devient le lieu de la fraternité, et lui-même renoue avec ses origines illustres. Avec la complicité de l'Eglise il revendique l'héritage d'Edward III «en toute justice» (I. ii. 96), et prend sa place dans une lignée de héros qui fait oublier le regrettable détour par l'usurpation : on ne parle pratiquement plus d'Henry IV, mais du Prince Noir, le vainqueur de Crécy, et de son père, l'immense Edward III,

his mountain sire, on mountain standing, Up in the air, crown'd with the golden sun, 
Henry V, «a stem / Of that victorious stock» (II. iv. 62-63), confirme son origine en ajoutant un épisode glorieux à leurs exploits, et pour plus de garantie, il en commence lui-même le récit :

This story shall the good man teach his son ; And Crispin Crispian shall ne'er go by, From this day to the ending of the world, But we in it shall be remembered;

(IV. iii. 56-59)

Aux histoires tristes de ses prédécesseurs il substitue l'histoire exaltante des grandes batailles, la légende héroïque.

D'autre part il justifie personnellement son accession à la dignité royale par l'empire qu'il a d'abord acquis sur lui-même :

We are no tyrant, but a Christian king;

Unto whose grace our passion is as subject

As is our wretches fetter'd in our prisons :

(I. ii. 241-243)

Ce titre est également consacré par l'Eglise : il s'est réformé instantanément à la mort de son père,

Consideration like an angel came,

And whipp'd th'offending Adam out of him, Leaving his body as a Paradise, T'envelop and contain celestial spirits.

(I. i. 28-31)

C'est ainsi qu'Henry $\mathrm{V}$ parvient à réconcilier la loi morale et les impératifs politiques : en institutionnalisant la séparation entre la personne humaine et la Couronne, il fait de la vertu une affaire privée, individuelle, sans relation aucune avec les exigences du service public. Le roi n'est qu'un être humain comme les autres, responsable du salut de son âme, dont la position éminente lui impose de pratiquer une vertu exemplaire et le pardon des offenses 
à titre privé. La dignité royale est une machine impersonnelle, inhumaine, qui n'admet aucune forme de 1èse-majesté :

Touching our person seek we no revenge ; But we our kingdom's safety must so tender, Whose ruin you have sought, that to her laws We do deliver you.

(II. iii. 174-177)

Le soldat qui l'a défié sans le connaître est innocent, les conjurés sont exécutés. Ce n'est plus l'outrage à l'oint du Seigneur, c'est l'atteinte à la sécurité de l'Etat qui devient sacrilège. La rébellion est désignée comme «Another fall of man» (II. ii. 141-142), mais les connotations religieuses renvoient à une doctrine laïque d'obéissance, fondée sur la raison d'Etat, et cautionnée par une Eglise soucieuse de défendre ses biens terrestres.

Dans cet univers déchu, Henry $V$ établit le pouvoir sur trois piliers de sagesse politique : sa conduite irréprochable, la gloire de ses ancêtres, et la religion d'Etat. Conscient du pouvoir des mythes il entreprend de les gérer lui-même en faisant glisser le culte de la majesté sacrée, du lieutenant de Dieu, à l'institution monarchique.

Henry $V$ réussit parce qu'il ne commet aucune des fautes de ses prédécesseurs. Richard II avouait dans sa prison : «I wasted time, and now doth time waste me;» (V. v. 49) ; Henry V lui, «weighs time / Even to the utmost grain.» (II. iv. 137-138). Il conserve juste ce qu'il faut de la tradition ": le récit des origines, la légende héroïque et la liturgie, mais tout indique qu'il s'est affranchi des exemples du passé : le capitaine gallois insiste pour qu'on fasse la guerre comme la faisaient les Romains; Henry V le trouve courageux, certes, «Though it appear a little out of fashion» (IV. i. 83). Henry est peut-être l'émule des anciens héros mais il n'est pas Alexander the Pig, et Monmouth ne ressemble pas tant que ça à la Macédoine.

Le roi qui meurt lentement tout au long de l'histoire 
des Plantagenets, c'est le roi divin. Le fonds mythique des histoires tristes de Shakespeare, c'est sa disparition et sa difficile renaissance. La majesté sacrée finit par renaître par la volonté d'Henry $\mathrm{V}$, mais c'est devenu un my the d'une autre nature, «une construction logique pour résoudre une contradiction». On parvient alors au terme d'un long processus d'intériorisation. Les personnages de la première tétralogie croyaient aux présages, ils étaient constamment côtoyés par des puissances surnaturelles ; Richard III qui niait toute transcendance était assailli par des fantômes. Dans la seconde série, quand Glendower parle des phénomènes qui ont présidé à sa naissance, il ne rencontre qu'un scepticisme amusé (1H4, III. i). Sous le règne d'Henry $\mathrm{V}$, «miracles are ceas'd» (I. i. 67), et chaque homme porte en lui-même son paradis - ou son enfer. Si-Henry attribue à Dieu tout le mérite de sa victoire, c'est une façon de souligner qu'il a obtenu l'approbation divine (IV. viii. 116-118).

Jean-Pierre Vernant explique que la tragédie fait son apparition à Athènes à un moment particulier de l'histoire de la cité,

celui où une distance s'est creusée au coeur de l'expérience sociale, assez grande pour qu'entre la pensée juridique et politique d'une part, les traditions mythiques et héroïques de l'autre, les oppositions se dessinent clairement, assez courte cependant pour que les conflits de valeur soient encore douloureusement ressentis et que la confrontation ne cesse pas de s'effectuer. ${ }^{19}$

Ce que dramatisent les deux tétralogies, c'est très précisément ce moment historique en Angleterre, celui où on commence à considérer le mythe avec les yeux du citoyen ; le moment où les liens avec le passé mythique commencent à se distendre, sans être encore rompus. C'est la fin de la relation magique entre le roi, la nature, et la divinité, et sa reconstitution synthétique par un gouvernant adroit. A l'inverse de ce qui se produit généralement, au lieu de dis- 
soudre l'événement dans une catégorie exemplaire, le my the prend une signification historique précise : le monstrueux Richard III est un produit de la guerre civile, et l'épopée d'Henry V une création de l'Etat moderne.

$\mathrm{La}$ conscience historique qui se constitue lentement au cours du XVIe siècle finira par briser le cercle magique de l'éternel retour ; en développant le sens du contexte elle va remettre en question la valeur de l'analogie entre présent et passé dans les modèles éducatifs, et bousculer les autorités traditionnelles. Elle va surtout modifier profondément la perception du temps, le temps aboli des mythes, et le temps immobile du Moyen Age chrétien.

Avec un sens historique souvent plus aigu que celui de ses contemporains, Shakespeare perçoit que le présent est enraciné dans le passé mais qu'il ne le reproduit pas. La leçon de l'histoire est sensible dans tout son théâtre, dominé par la course vectorisée du temps et le sens de de l'irréversible. C'est cette perception nouvelle du temps qui informe sa vision tragique c'est l'impossibilité de remonter le cours du temps qui crée l'irréparable, qui fait le caractère unique de chaque événement et de chaque individu.

Les pièces historiques ne constituent pas vraiment un genre à part. Toutes les pièces de Shakespeare représentent l'accomplissement d'un processus qui exclut le retour en arrière : l'ordre ancien fait place à l'ordre nouveau, et demain ne peut rattraper hier. La version tragique est plus près de l'histoire, elle met l'accent sur le prix dont se paie cette révolution, les pertes irréparables qu'entraîne la disparition de l'ordre ancien ; la comédie, plus proche du mythe, insiste sur la promesse de renouveau, sur l'action des forces vives qui régénèrent la nature. $\mathrm{La}$ comédie n'est pas plus irréaliste que la tragédie, elle fait intervenir un autre plan de réalité : elle exprime quelque chose qui est de l'ordre du désir, peutêtre une prière, ou un acte de foi. Vernant,

Même chez les plus optimistes des Tragiques, dit,

l'exaltation de l'idéal civique, l'affirmation de sa victoire sur toutes les forces du passé 
ont moins le caractère d'un constat, d'une tranquille assurance, que d'un espoir et d'un appel, où l'angoisse ne cesse jamais d'être présente, même dans la joie des apothéoses finales. 20

Les deux tétralogies s'achèvent au milieu d'un tas de cadavres, par un mariage, une réconciliation, et la promesse d'une société nouvelle. Elles sont tendues, elles aussi, entre le mythe et 1'histoire, entre deux exigences fondamentales de l'être humain : la stabilité politique, et la dimension spirituelle de l'existence.Car au fond la force du mythe,c'est d'imposer à l'histoire la matière de nos rêves.

Dominique GOY-BLANQUET. 


\section{NOTES}

1. W. Smith Fussner, The Historical Revolution, Westport, Conn., 1976 , p. 59.

2. W. Smi th Fussner, op. cit. , p. 46.

3. Northrop Frye, Fables of Identity, New York, 1963, p. 46.

4. Northrop Frye, Anatomy of Criticism, Princeton, 1957.

5. Mirce a Eliade, Aspects du Mythe, Paris, 1963.

6. Claude Lévi-Strauss, An thropologie Structurale, Paris, 1974. p. 119.

7. Northrop Frye, A Natural Perspective, Columbia U. P., 1965,

8. Louis-Vincent Thomas, Anthropologie de la Mort, Paris, 1975, pp. 433-452, qui cite C. Lévi-Srauss.

9. Qaude Lévi-Strauss, op. cit, , pp. 199-226.

10. Aristote, La Poétique, trad. R. Dupont-Roc et J. Lallot, Paris, 1980 , ch. 14, 53 b 19.

11. Aristote, op. cit., ch. 14,54 a 9.

12. Northrop Frye, Fables of Identity, pp. 46-47.

13. James G. Frazer, Le Rameau d'Or, "Le Roi Magicien dans la Société Primitive»et «Le Dieu qui meurt», trad. Perre Sayn, Paris 1981, 1983.

14. Luc de Heusch et al. Le Pouvoir et le Sacré, Bruxelles, 1962, Introduction par Armand Abel, p. 11.

15. Luc de Heusch, Le Roi Ivre ou l'Origine de l'Etat, Paris, 1972.

16. Mircea Eliade, Le Mythe de l'Etemel Retour, Paris, 1969.

17. Ge orges Dumézil, Mythe et Epopée, vol. I, Paris, 1968.

18. Edward I. Berry, Patterns of Decay, Shake speare's Early Histories, Virginia U.P., 1975, p. 95.

19. Jean-Pierre Vernant, «Le moment historique de la tragédie», dans Mythe et Tragédie en Grèce Ancienne, Paris, 1973, p. 16.

20. Jean-Pierre Vernant, «Tensions et ambiguìtés dans la tragédie», op. cit., p. 25. 